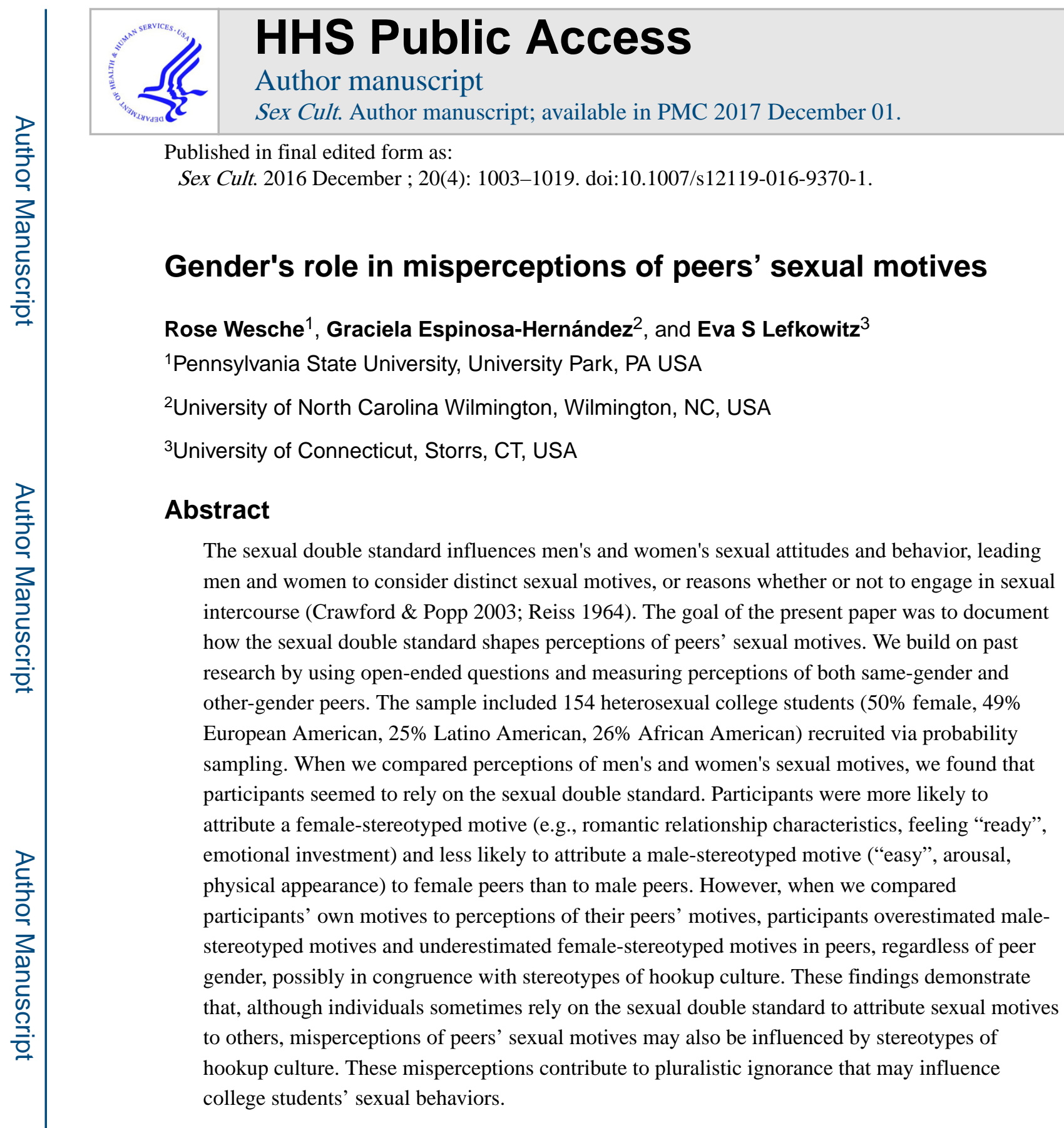

Research on sexual motives highlights gender differences, consistently finding that men and women consider different reasons for having or not having sexual intercourse (Cooper et al. 1998; Davis et al. 2004; Meston and Buss 2007). Gender differences in sexual attitudes and behavior are influenced by the sexual double standard, which refers to traditional gender socialization that encourages men to enjoy their sexuality and take active roles in sexual

Correspondence to: Rose Wesche.

Compliance with Ethical Standards

Conflict of Interest: The authors declare that they have no conflict of interest.

Ethical approval: All procedures performed in studies involving human participants were in accordance with the ethical standards of the institutional and/or national research committee and with the 1964 Helsinki declaration and its later amendments or comparable ethical standards. This article does not contain any studies with animals performed by any of the authors.

Informed consent: Informed consent was obtained from all individual participants included in the study. 
interactions, while encouraging women to take passive or reactive roles and to suppress sexual desire (Baumeister and Twenge 2002; Crawford and Popp 2003; Reiss 1964). Gendered socialization leads men and women to consider distinct sexual motives, or reasons whether or not to engage in sexual intercourse (Elliot 2006; Impett et al. 2005). For instance, men are more likely than women to express physical pleasure as a sexual motive (Cooper et al. 1998; Davis et al. 2004; Meston and Buss 2007). In contrast, women are more likely to consider relationship issues and intimacy as sexual motives (Cooper et al. 1998; Meston and Buss 2007). In addition to organizing one's own sexual motives, the sexual double standard may influence perceptions of same- and other-gender peers' sexual motives. It is important to understand how the sexual double standard shapes not only individuals' own sexual motives, but also perceptions of others' motives, because men and women may change their own sexual behaviors to align with perceived norms among peers and partners (Martens et al. 2006; Scholly et al. 2005). Misperceiving peers' and partners' sexual motives may therefore contribute to the perpetuation of the sexual double standard and miscommunication in sexual encounters.

Limited research has examined how the sexual double standard contributes to misperceptions of peers' sexual motives. The goal of the present paper is to document how the sexual double standard shapes perceptions of one's own and peers' sexual motives. We build on past research by using open-ended rather than forced choice questions about sexual motives. In addition, we incorporate gender into our analysis, distinguishing between perceptions of same-gender and other-gender peers. This distinction is a rudimentary way to separate perceptions of peers and potential sexual partners among heterosexual individuals, furthering understanding of the contributions of gender and the sexual double standard to sexual motives. The sexual double standard is based on a heterosexual model, and thus, we draw from a heterosexual model in interpreting our findings. Although research is beginning to expand understanding of the sexual double standard to sexual minority populations-for example, the sexual double standard may extend to perceptions of gay and lesbian peers' sexual behavior (Zaikman et al. 2016) — it is unknown how one's own sexual orientation may influence perceptions of peers' sexual motives. We acknowledge that the distinction between male/female peers does not directly translate to friends versus sexual partners for either heterosexual or sexual minority individuals.

\section{The Sexual Double Standard}

The sexual double standard refers to the phenomenon in which women are encouraged to suppress sexual desire and have few sexual partners, whereas men are permitted to enjoy their sexuality, take active roles in sexual interactions, and have many sexual partners (Baumeister and Twenge 2002; Crawford and Popp 2003; Milhausen and Herold 1999; Reiss 1964). Both evolutionary and cultural explanations exist for the sexual double standard, respectively highlighting biological and social reasons why differing expectations exist for men's and women's sexual behaviors and motives. The evolutionary perspective argues that men and women evolved to face different reproductive pressures. Men have an evolutionary advantage to reproducing with as many partners as possible, whereas women's labor-intensive role in childrearing is aided by being selective about sexual partners and choosing partners with whom they share an emotional connection (Feingold 1992; Oliver 
and Hyde 1993; Trivers 1972). Therefore, women gain an evolutionary advantage by being motivated by intimacy and considering relational aspects of their sexual partnerships, whereas men gain an evolutionary advantage by being motivated by physical pleasure and partner's appearance. Cultural explanations of the sexual double standard take a more social constructionist stance, positing that women are socialized to be sexually passive and adopt the role of sexual gatekeeper, whereas society does not place the same restrictions on men's sexuality (Averett et al. 2008; Wiederman 2005). Cultural restrictions on women's sexuality also mean that women are stigmatized for having casual sex or having too many sexual partners (Baumeister and Twenge 2002; Crawford and Popp 2003).

\section{Sexual Motives}

The sexual double standard likely contributes to gender differences in sexual motives. Although many men and women report being motivated by both intimacy and physical pleasure in their sexual relationships (Cooper et al. 1998; Meston and Buss 2007), research consistently finds gender differences in sexual motives. Men are more likely than women to endorse physical pleasure motives, whereas women are more likely than men to endorse intimacy as a sexual motive (Cooper et al. 1998; Meston and Buss 2007).

In addition to shaping one's own sexual motives, the sexual double standard likely shapes perceptions of peers' sexual motives. Misperceiving peers' sexual motives according to gender stereotypes may have consequences for sexual decision-making. Social norms theory suggests that individuals align their sexual behaviors to perceived social norms (Martens et al. 2006; Scholly et al. 2005). Individuals who perceive that normative sexual motives among peers align with the sexual double standard may act in accordance with the sexual double standard themselves. Similarly, individuals who expect a partner to behave according to the sexual double standard may alter their own behavior to account for expected behavior from a partner.

Perceptions of others' motives may be especially relevant to sexual behavior in college contexts because college students are likely to have new or casual sexual partners. College students may be likely to have new sexual partners for two reasons. First, achieving romantic and sexual competence is a key task of emerging adulthood (Arnett 2000), and sexual exploration is important to achieving this goal. Second, college campuses are characterized by hookup culture, which encourages participation in casual sexual encounters (Heldman and Wade 2010). In sexual interactions with new or casual partners, individuals may have limited knowledge about their partners' actual sexual motives because they have little experience with the partner or because of a lack of communication about sexual and relationship issues (Paul 2006; Paul and Hayes 2002). Because knowledge of partners' actual sexual motives is low, individuals with new or casual sexual partners must rely more on perceptions of motives in determining behavior. Given the implications for college students' sexual communication and behavior, it is important to understand perceptions of peers' sexual motives. 


\section{Perceptions of Men's and Women's Motives}

In this paper, we test the possibility that perceptions of peers' sexual motives align with the sexual double standard. We build on past research by distinguishing between perceptions of same-gender and other-gender peers, because gender is a salient characteristic individuals use to organize and make sense of their immediate environments (Bem 1974). We focus on three comparisons: differences in perceived sexual motives of male peers versus female peers, misperceptions of same-gender peers' motives, and misperceptions of other-gender peers' motives. First, we examine perceptions of male peers' versus female peers' sexual motives. Because this analysis does not compare perceived motives to actual motives, it does not test for the presence of a stereotype. Rather, we look to replicate past research that has found evidence of a sexual double standard in perceptions of sexual motives (Davis et al. 2004; Meston and Buss 2007; Schachner and Shaver 2007). We hypothesize that perceptions of peers' motives will be congruent with the sexual double standard. Therefore, we predict that emerging adults will report that female peers are more likely to consider femalestereotyped sexual motives and less likely to consider male-stereotyped sexual motives than male peers are (Hypothesis 1).

In addition to examining differences in perceptions of male peers' versus female peers' sexual motives, we compare self-reported sexual motives to perceptions of peers' sexual motives. One of these comparisons focuses on differences between participants' own sexual motives and perceptions of same-gender peers' motives (e.g., how female participants' own sexual motives differ from the motives they attribute to a typical female student at their university). Individuals tend to view others as more stereotypical than themselves because they have more information on themselves, and rely on stereotyping of others in the absence of additional information (Doosje et al. 2007; Taylor et al. 1978; Wittenbrink et al. 1998). Because individuals tend to stereotype others, we hypothesize that they may rely on the sexual double standard more in describing peers' sexual motives than their own. Thus, their perceptions of peers' motives may be more concordant with the sexual double standard than their own motives. In other words, male participants will perceive their male peers to be more likely to consider male-stereotyped sexual motives and less likely to consider femalestereotyped sexual motives than they are themselves (Hypothesis 2A). Female participants will perceive their female peers to be more likely to consider female-stereotyped sexual motives and less likely to consider male-stereotyped sexual motives than they are themselves (Hypothesis 2B).

In addition to comparing self-reported motives to perceptions of same-gender peers' motives, we compare emerging adults' perceptions of other-gender peers' motives to othergender peers' self-reported motives. For example, we compare the sexual motives that male participants attribute to a typical female student at their university to the self-reported sexual motives of female participants in our sample. This comparison may help us understand how heterosexual emerging adults perceive potential sexual partners. Past research has demonstrated that both men and women misperceive other-gender peers' sexual behaviors and attitudes. For instance, men consistently rate women as behaving more sexually (e.g., flirtatious) than women rate themselves (Abbey et al. 2000; Edmondson and Conger 1995). Similarly, men and women attribute riskier sexual behavior to other-gender peers than their 
other-gender peers actually report (Lewis et al. 2007). However, to our knowledge, it is unknown whether men's and women's perceptions of other-gender peer motives align with the sexual double standard or not. We predict that both men and women will genderstereotype their other-gender peers (Hypothesis 3).

In summary, we had the following questions and hypotheses:

1. How do emerging adults' perceptions of the sexual motives of female and male peers differ? We hypothesize that participants will be more likely to attribute male-stereotyped motives and less likely to attribute femalestereotyped motives to male peers than to female peers.

2.

How do emerging adults' own sexual motives differ from their perception of same-gender peers' motives?

2A) Male participants will be more likely to attribute a malestereotyped sexual motive (e.g., sexual arousal) and less likely to attribute a female-stereotyped motive (e.g., emotional involvement) to their male peers than to themselves.

2B) Female participants will be less likely to attribute a malestereotyped motive and more likely to attribute a femalestereotyped motive to their female peers than to themselves.

3.

How do emerging adults' perceptions of other-gender peers' motives differ from other-gender peers' own self-reported motives?

3A) Male participants will be less likely to attribute a malestereotyped sexual motive and more likely to attribute a femalestereotyped motive to female peers than female participants report for themselves.

3B) Female participants will be more likely to attribute a malestereotyped motive and less likely to attribute a femalestereotyped motive to male peers than male participants report for themselves.

These three analytic questions allow us to measure the sexual double standard in different ways - specifically, we can examine perceptions of others (question 1), and we can compare one's own motives to perceptions of peers' motives (questions 2 and 3). In order to perform the comparisons described above, we asked heterosexual emerging adults about their own sexual motives, and perceptions of same-gender and other-gender peers' sexual motives. Some studies have assessed motives with standardized scales (Cooper et al. 1998; Leigh 1989; Ozer et al. 2003), whereas other researchers have chosen open-ended formats to assess these motives (Allen et al. 2008; Michels et al. 2005). Standardized scales (Cooper et al. 1998) are useful for providing consistency across participants. We chose an open-ended assessment because we wanted to allow participants to describe sexual motives in their own words without constraining responses to a limited number or certain type of motive. Openended responses capture the motives emerging adults consider to be important for themselves and for their peers. Using questions about same-gender and other-gender peers 
allows us to understand how perceptions of peers' sexual motives shape the social landscape and may contribute to sexual miscommunication, advancing understanding of sexual motives beyond comparisons of men and women's self-perceptions.

\section{Method}

\section{Participants}

Data for this study come from the first (T1, Fall semester of first year of college) and third (T3, Fall semester of second year) time points in a longitudinal study of college students' sexual health. At T1, we recruited first year college students from a large Northeastern university to participate. We contacted all African American and Latino American first year students between the ages of 17 and 19, and a randomly selected subsample of European American students (9\% of all students age 17 - 19 who were Caucasian, not Hispanic according to the Registrar's definition). From the initial 839 students invited to participate, $52 \%$ agreed for a total of 434 participants. At each time point, the study consisted of two sessions: a survey phase and a video phase. For the survey phase, we contacted participants by e-mail or phone to schedule an appointment. Participants completed paper and pencil surveys in groups. All students completed informed consent. After participating in the survey phase at $\mathrm{T} 1$, a random sub-sample of participants $(N=182)$ was invited by e-mail or phone to participate in the video phase. We excluded participants who reported being exclusively lesbian/gay (less than 1\%) from the $\mathrm{T} 1$ video phase, because we were interested in assessing gendered behaviors in mixed gender interactions where physical/sexual attraction may exist. During this video phase, participants engaged in videotaped tasks in male-female pairs (videotaped data not used in current analyses), and then filled out surveys in separate rooms. At T3, participants received $\$ 35$ for the survey phase and $\$ 25$ for the video phase. In this paper, we draw demographic information from the T1 survey phase and open-ended data (self and peer motives) from the survey in the $\mathrm{T} 3$ video phase.

The current sample consists of 154 (50\% female) second year heterosexual college students, age 18.9 to 20.8 at T3 $(M=19.6, S D=0.4)$. Twenty six percent of the participants were African American, 25\% were Latino American, and 49\% were European American. Seventy percent of the participants were sexually experienced (i.e., had had penetrative sex) by T1.

The retention rate from the $\mathrm{T} 1$ video phase to the $\mathrm{T} 3$ video phase was $81 \%$. Of the students who completed the T1 video phase but did not return for the T3 video phase $(n=35), 80 \%$ were no longer eligible to participate, mainly because they were no longer enrolled in the university or were on leave $(n=27)$. Moreover, there were 7 students who participated in the video phase at $\mathrm{T} 3$, but at $\mathrm{T} 1$ had participated in the survey phase but not the video phase.

We examined any potential biases due to selection of the video phase subsample and attrition by comparing the demographic information of participants who completed surveys at the T3 video phase $(N=154)$ to all other participants in the larger T1 study $(N=280)$ on demographics using three chi-square tests and one t-test. These two groups did not significantly differ on gender or sexual experience at T1. A chi-square test indicated differences in race/ethnicity between the two groups $\left(\chi^{2}(2)=11.51, p<.01\right)$, with participants in the $\mathrm{T} 3$ video phase more likely to be European American and less likely to be 
African American than other participants. Additionally, participants in the T3 video phase were older $(t(432)=-2.69, p<0.01$.

\section{Measures and Procedure}

We used open-ended questions to assess sexual motives. This section of the survey began as follows: "When deciding to become sexually intimate with someone, people may consider different things such as personal beliefs, partner's characteristics, how well people know each other or the situation, to name a few." Participants then answered three open-ended questions about self-motives, same-gender peer motives, and other-gender peer motives. The specific three questions were: "What do you consider necessary/most important when deciding to have sex with someone?"; "What do you think a male student at [name of the university] considers necessary/most important when deciding to have sex with someone?"; "What do you think a female student at [name of the university] considers necessary/most important when deciding to have sex with someone?" Participants always answered the selfmotives question first. To control for order effects, half of the participants answered the female student question second, and the other half answered the male question second.

Coding of themes-Based on past research (e.g., Cooper et al. 1998; Leigh 1989; Meston and Buss 2007; Ozer et al. 2003) and participants' responses, we developed a list of themes to classify responses to the three questions. The coding system was identical for each of the open-ended questions to allow comparisons across the three targets (self, female student, and male student). The coding system consisted of 22 codes. Finally, we included the option "uncodeable" for responses that could not be coded due to circumstances such as illegible handwriting or failing to answer the question (e.g., the response was left blank).

Three undergraduate students coded all responses for the presence or absence of general categories and subcategories. Coders met with the second author twice a week for an hour on average to discuss discrepancies and make final coding decisions (codes based on these final decisions were used in all analyses). Codes were not mutually exclusive in that responses could be coded into more than one category. We performed pairwise kappas to assess inter-rater reliability. We computed three kappas (one for each coder pair), and calculated the mean across the three kappas for each category. In Table 1, we report the ranges and averages across the 3 coder pairs. The average kappa was above .80 for all codes.

Code selection-For the current analyses, we selected codes that corresponded to stereotypically male or female sexual motives (see Table 1). For analysis, we created six composite dichotomous variables for each participant indicating whether the participant reported perceiving any male-stereotyped or any female-stereotyped motive for her/himself, female peers, or male peers (see Table 2). As an example of a male-stereotyped motive, one male participant, when asked about male peers' motives, wrote that his male peers wanted a "hot chick, hot body"- a physical appearance motive. As an example of a femalestereotyped motive, one female participant wrote that her female peers wanted "some kind of emotional bond"-an emotional investment motive. 


\section{Results}

Before testing our hypotheses, we were interested to see whether there were gender differences in reporting male- and female-stereotyped sexual motives for oneself, which would be consistent with past research (Carroll et al. 1985; Davis et al. 2004; Meston and Buss 2007; Schachner and Shaver 2007). We performed this comparison using two chisquare analyses. Male participants were more likely to report a male-stereotyped self-motive than female participants were $\left(X^{2}(1)=14.70, p<.001\right)$. Female participants were more likely to report a female-stereotyped sexual motive than male participants were $\left(X^{2}(1)=\right.$ 9.66, $p<.01)$.

Hypothesis 1 was that participants would be more likely to attribute male-stereotyped motives and less likely to attribute female-stereotyped motives to male peers than to female peers. To test this hypothesis, we performed two repeated measures ANOVA's with dependent variables of male-stereotyped and female-stereotyped motives, and peer gender (female peer vs. male peer) as a within-subjects factor. Participants were more likely to attribute a male-stereotyped motive to male peers than to female peers $(F(1,153)=28.53, p$ $<.001$; proportions of male and female participants' attributions are reported in Table 2), and more likely to attribute a female-stereotyped motive to female peers than male peers ( $F$ $(1,153)=66.01, p<.001 ;$ proportions reported in Table 2). Thus, Hypothesis 1 was supported. Hypothesis 2 was that (2A) male participants would be more likely to attribute a male-stereotyped motive and less likely to attribute a female-stereotyped motive to their male peers than to themselves, and that (2B) female participants would be less likely to attribute a male-stereotyped motive and more likely to attribute a female-stereotyped motive to their female peers than to themselves.

We tested these hypotheses using four repeated-measures ANOVAs comparing self-reported sexual motives to perceptions of same-gender peers' sexual motives. In these analyses, malestereotyped and female-stereotyped sexual motives were dependent variables and the withinsubjects factor was whether the participant was reporting his/her own motives or perceptions of same-gender peers' motives. Separate analyses were conducted for male and female participants. In each ANOVA, there was a statistically significant main effect of self vs. same-gender peer. Both male and female participants were more likely to attribute a malestereotyped motive to same-gender peers than to themselves (men: $F(1,76)=21.64, p<$. 001 ; women: $F(1,76)=20.25, p<.001)$. Additionally, both male and female participants were less likely to attribute a female-stereotyped sexual motive to same-gender peers than to themselves (men: $F(1,76)=70.66, p<.001$; women: $F(1,76)=70.99, p<.001$; see Table 2 for proportions). That is, male participants misperceived their same-gender peers' sexual motives in a manner congruent with sexual double standard beliefs, but female participants' misperceptions of their same-gender peers' sexual motives did not correspond to the sexual double standard. Thus, Hypothesis 2A was supported, but 2B was not.

Hypothesis 3 was that (3A) male participants would be less likely to attribute a malestereotyped sexual motive and more likely to attribute a female-stereotyped motive to male peers than female participants report for themselves, and that (3B) female participants would be more likely to attribute a male-stereotyped motive and less likely to attribute a female- 
stereotyped motive to male participants than male participants report for themselves. To test these hypotheses, we performed four one-way between-subjects ANOVAs comparing participants' own self-reported motives to other-gender participants' perceptions of their sexual motives. That is, we compared female participants' own self-reported motives to male participants' perceptions of their female peers' motives, and we compared male participants' own self-reported motives to female participants' perceptions of their male peers' motives. In these analyses, male-stereotyped and female-stereotyped sexual motives were dependent variables. Separate analyses were conducted by gender, for four total tests. We found that female participants perceived their male peers as more likely to consider male-stereotyped sexual motives $(F(1,152)=8.68, p<.01)$ and less likely to consider female-stereotyped sexual motives $(F(1,152)=34.46, p<.001)$ than male participants actually reported (see Table 2 for proportions). Male participants perceived female peers as more likely to consider male-stereotyped sexual motives $(F(1,151)=70.07, p<.001)$ and less likely to consider female-stereotyped sexual motives $(F(1,152)=159.21, p<.001)$ than female participants actually reported (see Table 2 for proportions). That is, female participants misperceived men's sexual motives in a manner congruent with sexual double standard beliefs, but male participants' misperceptions of women's sexual motives did not correspond to the sexual double standard. Thus, Hypothesis 3B was supported, but 3A was not.

\section{Discussion}

The purpose of this paper was to examine whether college students' perceptions of peers' gender-stereotyped sexual motives aligned with the sexual double standard, building on past research by using open-ended questions about sexual motives and distinguishing between perceptions of same-gender and other-gender peers. The results suggest that, under some conditions, college students' sexual motives are consistent with the sexual double standard. However, some comparisons suggested that individuals misperceive both men's and women's sexual motives in the same way-they overestimate their peers' male-stereotyped motives and underestimate their peers' female-stereotyped motives, regardless of their own gender. These findings raise questions about the extent to which the sexual double standard influences perceptions of sexual motives and provokes consideration of what other cognitions might influence perceptions of peers' sexual motives.

When we simply examined perceptions of one's own motives, or compared students' perceptions of female peers to male peers, individuals seemed to rely on the sexual double standard. Men were more likely than women to report a male-stereotyped motive and less likely to report a female-stereotyped motive, consistent with past research (Davis et al. 2004; Meston and Buss 2007; Schachner and Shaver 2007). Additionally, individuals were less likely to attribute a male-stereotyped motive and more likely to attribute a femalestereotyped motive to female peers than to male peers. These findings suggest that the sexual double standard influences college students' own sexual motives and perceptions of their peers' motives.

However, when we compared individuals' own self-reported motives to their perceptions of peers' motives, another pattern emerged. Whether comparing students' own motives to their perceptions of their same gender peers' motives, or comparing students' own motives to the 
other gender's perceptions of their motives, the findings were similar. Both men and women were more likely to attribute a female-stereotyped motive, and less likely to attribute a malestereotyped motive, to themselves than to others. These findings were contrary to our hypotheses, suggesting that the sexual double standard did not influence these comparisons. It is possible that, when comparing self to others, individuals rely more on stereotypes of college hookup culture than on the sexual double standard. Hookup culture stereotypes portray college students as highly motivated to engage in casual sex. Rather than looking to form an emotional connection with a partner, sexual scripts in hookup culture depict students as looking to experience physical pleasure and avoid commitment (Bogle 2008; Garcia et al. 2012; Paul 2006). Although arousal and appearance are traditionally malestereotyped motives, they are also consistent with the motives that stereotypically characterize college hookups, and students seem to misperceive these motives in a way that emphasizes peers' involvement in hookup culture.

Individuals' overestimation of their peers' male-stereotyped motives and underestimation of peers' female-stereotyped motives may be due to misperceptions of hookup culture. Past research has documented that college students misperceive their peers' acceptance of and involvement in hookup culture (Barriger and Vélez-Blasini 2013; Lambert et al. 2003). Although individuals perceive that their peers often engage in casual sexual behavior, the majority of college students' sexual activity occurs in the context of romantic relationships (Fielder et al. 2013). In fact, establishing intimate relationships is a key developmental task during emerging adulthood that is challenged by a cultural focus on casual sex (Shulman and Connolly 2013). Although college students believe that their peers often engage in casual sex, they know that they themselves are more likely to have sex in the context of a romantic relationship. These misperceptions of peers' behaviors may translate into misperceptions of sexual motives, causing individuals to perceive their peers as more motivated by physical appearance, arousal, and "easy" sexual encounters, and less motivated by emotions, compatibility, and other relationship factors.

Additionally, because of the importance of emotional intimacy during emerging adulthood, individuals may believe that intimacy-focused motives are more legitimate or desirable than the physical appearance- and pleasure-focused motives that stereotypically characterize casual sex. Social comparison theory posits that individuals tend to attribute more positive, and fewer negative, qualities to themselves than to others in an attempt to preserve selfesteem (Bradley 1978; Festinger 1954; Stapel and Blanton 2007). Therefore, they may use the stereotypes of "shallow" hookup culture for others, but portray themselves as motivated by intimacy.

In addition to the possibility that misperceptions of peers' sexual motives correspond to misperceptions of hookup culture, our findings can be interpreted in the context of recent evidence of a reverse double standard. Our findings differ from the results of several recent studies finding that, for some individuals and under some circumstances, men's sexual activity is perceived more negatively than women's, or that women with many sexual partners are perceived positively (Howell et al. 2011; Milhausen and Herold 1999; Milhausen and Herold 2002; Papp et al. 2015; Zaikman et al. 2014). We extend this body of literature by examining perceptions of sexual motives, whereas past research on a reverse 
sexual double standard has examined perceptions of sexual behavior. A reverse double standard in perceptions of sexual motives would predict that individuals overestimate male peers' female-stereotyped motives and underestimate male peers' male-stereotyped motives, whereas the reverse would be true for perceptions of female peers. Our finding that individuals overestimate both male and female peers' male-stereotyped sexual motives and underestimate their female-stereotyped sexual motives is not consistent with a reverse sexual double standard, which would still expect gender differences in misperceptions of sexual motives. Rather, it suggests that gender may not be as crucial to self-other comparisons of sexual motives as past research suggests.

Whether our findings on perceptions of peers versus self are due to overestimation of hookup culture or some other explanation, they highlight the fact that the sexual double standard does not always influence perceptions of others' sexual motives. This finding may inform evolutionary and cultural understandings of the sexual double standard. Evolutionary explanations of the emergence of the sexual double standard, which propose that the sexual double standard emerged from biologically-driven gender differences, would suggest that individuals misperceive others' sexual motives in accordance with the sexual double standard consistently across circumstances. However, our finding that perceptions of peers' sexual motives do not always adhere to the sexual double standard emphasizes the importance of cultural and social factors in explaining the sexual double standard. Researchers have argued that changing personal and cultural expectations about sexuality may lead to rejection of a traditional sexual double standard (Farvid et al. 2016; Jackson and Cram 2003; Milhausen and Herold 1999). Prior research supporting this claim has found inconsistent evidence of the sexual double standard (Bordini \& Sperb, 2013; Howell et al. 2011; Kreager et al. 2016; Marks and Fraley 2005; Milhausen and Herold 1999; Papp et al. 2015; Zaikman and Marks 2014). The present findings add to this literature, suggesting that the current social climate of hookup culture in emerging adulthood may shift perceptions of sexual motives away from a traditional gendered sexual double standard, and highlighting the role of social and cultural circumstances in explaining attributions related to others' sexual behaviors and sexual motives.

Our finding regarding perceptions of same-gender peers versus self has implications for perceptions of norms surrounding sexual encounters. If individuals believe that their peers are more motivated by physical attraction and pleasure and less motivated by relationship characteristics and emotions than they are themselves, it contributes to an environment of pluralistic ignorance, in which individuals believe that their private attitudes or beliefs are discrepant from the norm (Allport 1924; Allport 1933; Lambert et al. 2003). Although individuals privately reject a norm of sexual behavior driven by physical attraction rather than intimacy, if they incorrectly assume that others accept this norm, they are likely to adhere to it. Research on perceptions of sexual norms, as well as drinking norms, has found that overestimating peers' acceptance of these behaviors is associated with higher levels of involvement in these behaviors (Borsari and Carey 2001; Lewis et al. 2014; Lewis et al. 2007; Martens et al. 2006; Perkins et al. 2005). Individuals may feel pressure to act in accordance with what they perceive is the norm, which may mean engaging in more sexual behaviors than they are comfortable with, engaging in sexual encounters before they are ready, or focusing on the sexual aspects of a relationship while avoiding discussions about 
emotions or intimacy. Additionally, even individuals who act in accordance with their private sexual motives may feel a sense of alienation from their peers, or may feel reluctant to communicate with peers about sex, because they believe their motives are discordant with peers' motives.

Similarly, our finding regarding perceptions of other-gender peers' motives versus othergender peers' own self-reported motives has implications for emerging adults' sexual encounters. Men and women may misperceive their partners as more motivated by physical attraction and pleasure and less motivated by relationship characteristics and emotions than they really are. If partners rely on these misperceptions, they may not discuss relationship issues, compatibility, or whether they feel "ready" for sex with their partner. A lack of sexual communication in turn may correspond to negative outcomes, such as lower sexual satisfaction (Davis et al. 2006).

The findings highlight the fact that college students may use different strategies to compare men to women than to compare self to others. Although the sexual double standard still describes misperceptions of others' sexual motives to some extent, descriptive norms of sexual motives are determined by multiple social schemas and sexual scripts. This finding is consistent with research on norms for sexual behavior, which has found that students' perceptions of their peers' behavior are shaped by both gender and stereotypes of hookup culture (Lambert et al. 2003; Martens et al. 2006). Because of the complex cognitions underlying perceptions, the sexual double standard is not sufficient to describe misperceptions of others' sexual motives. Future research should examine how the sexual double standard and stereotypes of hookup culture interact with one another to influence perceptions of others' sexual motives.

The results also highlight the importance of analytic strategies in understanding perceptions of others. Previous research on sexual norms typically has examined perceptions of self versus other (Barriger and Vélez-Blasini 2013; Lewis et al. 2007; Lewis et al. 2014; Martens et al. 2006; for an exception, see Lambert et al. 2003). Although these social comparison analyses offer important behavioral implications, they are not the only way that emerging adults make sense of their peers' motives and behavior. The multiple strategies used to analyze perceptions of peers' motives in this paper allowed us to determine that different principles underlie perceptions of gender differences in sexual motives and perceptions of self versus other. Examining how individuals perceive men's motives versus women's motives may lead to different conclusions about how individuals perceive their peers, with additional implications for sexual behavior. Researchers should be mindful of how they measure perceptions, because they may receive different answers depending on whether the focus of analyses is on social comparison or gender differences.

Our findings must be interpreted in light of their limitations. First, we were interested in differentiating between same-gender and other-gender peers' sexual motives in order to distinguish peers and potential sexual partners. However, we asked participants about other men's and women's motives generally, not about motives of a friend and a potential sexual partner. This strategy assumes participants' heterosexuality, and further assumes that any other-gender peer is a potential sexual partner, both of which are flawed assumptions. 
Therefore, future research should consider directly asking about perceptions of friends and potential sexual partners. Second, even in sexual encounters, the sexual motives emerging adults consider in their own sexual decision-making may vary depending on the context. Emerging adults might report different sexual motives when they consider having a long term, as opposed to a short term, relationship with the sexual partner (Cooper et al. 1998).

In addition to its limitations, this study had several strengths. The open-ended nature of the measures allowed us to better understand sexual motives by allowing participants to identify which sexual motives they believe are important. Additionally, addressing our research question from several angles (male peers versus female peers, same-gender peers, and othergender peers) allowed us to examine the comparative roles of gender and self versus other comparisons in misperceptions of peers' sexual motives. Our findings raise questions about the salience of gender and the sexual double standard in attributions of peers' sexual motives. Emerging adults misperceived peers' sexual motives, sometimes (but not always) according to the sexual double standard. This finding highlights the need for peers and partners to communicate with each other about sexual motives in order to remedy misperceptions. The results of this paper can inform prevention programs that promote healthy sexual relationships by improving awareness of gender and hookup culture stereotypes in sexual decision-making, and encouraging communication of expectations between sexual partners.

\section{References}

Abbey A, Zawacki T, McAuslan P. Alcohol's effects on sexual perception. Journal of Studies on Alcohol. 2000; 61:688-697. [PubMed: 11022808]

Allen KR, Husser EK, Stone DJ, Jordal CE. Agency and error in young adults' stories of sexual decision making. Family Relations. 2008; 57:517-529.

Allport, EH. Social psychology. Houghton Mifflin; Boston: 1924.

Allport, FH. Institutional behavior. University of North Carolina Press; Chapel Hill: 1933.

Arnett JJ. Emerging adulthood: A theory of development from the late teens through the twenties. American Psychologist. 2000; 55:469-480. [PubMed: 10842426]

Averett P, Benson M, Vaillancourt K. Young women's struggle for sexual agency: The role of parental messages. Journal of Gender Studies. 2008; 17:331-344.

Barriger M, Vélez-Blasini CJ. Descriptive and injunctive social norm overestimation in hooking up and their role as predictors of hook-up activity in a college student sample. Journal of Sex Research 2013; 50:84-94. [PubMed: 21919644]

Baumeister RF, Twenge JM. Cultural suppression of female sexuality. Review of General Psychology. 2002; 6:166-203.

Bem SL. The measurement of psychological androgyny. Journal of Consulting and Clinical Psychology. 1974; 42:155-162. [PubMed: 4823550]

Borsari B, Carey KB. Peer influences on college drinking: A review of the research. Journal of Substance Abuse. 2001; 13:391-424. [PubMed: 11775073]

Bradley GW. Self-serving biases in the attribution process: A reexamination of the fact or fiction question. Journal of Personality and Social Psychology. 1978; 36:56-71.

Bogle, K. Hooking up: Sex, dating, and relationships on campus. York University Press; New York: 2008.

Bordini GS, Sperb TM. Sexual double standard: A review of the literature between 2001 and 2010. Sexuality \& Culture. 2013; 17:686-704.

Carroll JL, Volk KD, Hyde JS. Differences between males and females in motives for engaging in sexual intercourse. Archives of Sexual Behavior. 1985; 14:131-139. [PubMed: 3994500] 
Cooper ML, Shapiro CM, Powers AM. Motivations for sex and risky sexual behavior among adolescents and young adults: A functional perspective. Journal of Personality and Social Psychology. 1998; 75:1528-1558. [PubMed: 9914665]

Crawford M, Popp D. Sexual double standards: A review and methodological critique of two decades of research. The Journal of Sex Research. 2003; 40:13-26. [PubMed: 12806528]

Davis D, Shaver PR, Vernon ML. Attachment style and subjective motivations for sex. Personality and Social Psychology Bulletin. 2004; 30:1076-1090. [PubMed: 15257790]

Davis D, Shaver PR, Widaman KF, Vernon ML, Follette WC, Beitz K. "I can't get no satisfaction": Insecure attachment, inhibited sexual communication, and sexual dissatisfaction. Personal Relationships. 2006; 13:465-483.

Doosje B, Spears R, Redelijkheid H, Onna J. Memory for stereotype (in)consistent information: The role of in-group identification. British Journal of Social Psychology. 2007; 46:115-128. [PubMed: 17355721]

Edmondson CB, Conger JC. The impact of mode of presentation on gender differences in social perception. Sex Roles. 1995; 32:169-183.

Farvid P, Braun V, Rowney C. 'No girl wants to be called a slut!': Women, heterosexual casual sex and the sexual double standard. Journal of Gender Studies. Advance online publication. 2016 doi: 10.1080/09589236.2016.1150818.

Feingold A. Gender differences in mate selection preferences: A test of the parental investment model. Psychological Bulletin. 1992; 112:125-139. [PubMed: 1388281]

Festinger L. A theory of social comparison processes. Human Relations. 1954; 7:117-140.

Fielder RL, Carey KB, Carey MP. Are hookups replacing romantic relationships? A longitudinal study of first-year female college students. Journal of Adolescent Health. 2013; 52:657-659. [PubMed: 23298995]

Garcia JR, Reiber C, Massey SG, Merriwether AM. Sexual hookup culture: A review. Review of General Psychology. 2012; 16:161-176. [PubMed: 23559846]

Heldman C, Wade L. Hook-up culture: Setting a new research agenda. Sexuality Research and Social Policy. 2010; 7:323-333.

Howell JL, Egan PM, Giuliano TA, Ackley BD. The reverse double standard in perceptions of studentteacher sexual relationships: The role of gender, initiation, and power. The Journal of Social Psychology. 2011; 151:180-200. [PubMed: 21476461]

Impett EA, Peplau LA, Gable SL. Approach and avoidance sexual motives: Implications for personal and interpersonal well-being. Personal Relationships. 2005; 12:465-485.

Jackson SM, Cram F. Disrupting the sexual double standard: Young women's talk about heterosexuality. British Journal of Social Psychology. 2003; 42:113-127. [PubMed: 12713759]

Lambert TA, Kahn AS, Apple KJ. Pluralistic ignorance and hooking up. Journal of Sex Research. 2003; 40:129-133. [PubMed: 12908120]

Leigh BC. Reasons for having and avoiding sex: Gender, sexual orientation, and relationship to sexual behavior. Journal of Sex Research. 1989; 26:199-209.

Lewis MA, Lee CM, Patrick ME, Fossos N. Gender-specific normative misperceptions of risky sexual behavior and alcohol-related risky sexual behaviors. Sex Roles. 2007; 57:81-90.

Lewis MA, Litt DM, Cronce JM, Blayney JA, Gilmore AK. Underestimating protection and overestimating risk: Examining descriptive normative perceptions and their association with drinking and sexual behaviors. Journal of Sex Research. 2014; 51:86-96. [PubMed: 23067203]

Marks MJ, Fraley RC. The sexual double standard: Fact or fiction? Sex Roles. 2005; 52:175-186.

Martes MP, Page JC, Mowry ES, Damann KM, Taylor KK, Cimini MD. Differences between actual and perceived student norms: An examination of alcohol use, drug use, and sexual behavior. Journal of American College Health. 2006; 54:295-300. [PubMed: 16539222]

Meston CM, Buss DM. Why humans have sex. Archives of Sexual Behavior. 2007; 36:477-507. [PubMed: 17610060]

Michels TM, Kropp RY, Eyre SL, Halpern-Felsher BL. Initiating sexual experiences: How do young adolescents make decisions regarding early sexual activity? Journal of Research on Adolescence. 2005; 15:583-607. 
Milhausen RR, Herold ES. Reconceptualizing the sexual double standard. Journal of Psychology \& Human Sexuality. 2002; 13:63-83.

Milhausen RR, Herold ES. Does the sexual double standard still exist? Perceptions of university women. Journal of Sex Research. 1999; 36:361-368.

Oliver MB, Hyde JS. Gender differences in sexuality: A meta-analysis. Psychological Bulletin. 1993; 114:29-51. [PubMed: 8346327]

Ozer EJ, Dolcini MM, Harper GW. Adolescents' reason for having sex: Gender differences. Journal of Adolescent Health. 2003; 33:317-319. [PubMed: 14596952]

Papp LJ, Hagerman C, Gnoleba MA, Erchull MJ, Liss M, Miles-McLean H, Robertson CM. Exploring perceptions of slut-shaming on Facebook: Evidence for a reverse sexual double standard. Gender Issues. 2015; 32:57-76.

Paul, EL. Beer goggles, catching feelings, and the walk of shame: The myths and realities of the hookup experience.. In: Kirkpatrick, DC.; Duck, S.; Foley, MK., editors. Relating difficulty: The processes of constructing and managing difficult interaction. Lawrence Erlbaum; Mahwah, NJ: 2006. p. 141-160.

Paul EL, Hayes KA. The casualties of 'casual' sex: A qualitative exploration of the phenomenology of college students' hookups. Journal of Social and Personal Relationships. 2002; 19:639-661.

Perkins HW, Haynes MP, Rice R. Misperceiving the college drinking norm and related problems: A nationwide study of exposure to prevention information, perceived norms and student alcohol misuse. Journal of Studies on Alcohol. 2005; 66:470-478. [PubMed: 16240554]

Reiss IL. The scaling of premarital sexual permissiveness. Journal of Marriage and the Family. 1964; 26:188-198.

Schachner DA, Shaver PR. Attachment dimensions and sexual motives. Personal Relationships. 2004; 11:179-195.

Scholly K, Katz AR, Gascoigne J, Holck PS. Using social norms theory to explain perceptions and sexual health behaviors of undergraduate college students: An exploratory study. Journal of American College Health. 2005; 53:159-166. [PubMed: 15663064]

Shulman S, Connolly J. The challenge of romantic relationships in emerging adulthood: Reconceptualization of the field. Emerging Adulthood. 2013; 1:27-39.

Stapel, DA.; Blanton, H. Social identity and reference group comparisons.. In: Stapel, DA.; Blanton, H., editors. Social comparison theories: Key readings. Psychology Press; New York, NY: 2007.

Taylor SE, Fiske ST, Etcoff NL, Ruderman AJ. Categorical and contextual bases of person memory and stereotyping. Journal of Personality and Social Psychology. 1978; 36:778-793.

Trivers, R. Parental investment and sexual selection.. In: Campbell, B., editor. Sexual selection and the descent of man. Aldine; Chicago: 1972. p. 136-179.

Wiederman MW. The gendered nature of sexual scripts. The Family Journal. 2005; 13:496-502.

Wittenbrink B, Hilton JL, Gist PL. In search of similarity: Stereotypes as naive theories in social categorization. Social Cognition. 1998; 16:31-55.

Zaikman Y, Marks MJ. Ambivalent sexism and the sexual double standard. Sex Roles. 2014; 71:333344. 
Table 1

Proportions and Interrater Reliability for Self and Peer Motives

\begin{tabular}{lcccc}
\hline & Self & Female peer & Male peer & Kappas Range (Mean) \\
\hline Male-stereotyped motives & .13 & .29 & .51 & \\
Sexually aroused & .00 & .01 & .01 & $.87-.97(.89)$ \\
Easy & .00 & .00 & .03 & $.82-.91(.86)$ \\
Physical appearance & .13 & .29 & .49 & $.97-.98(.98)$ \\
Female-stereotyped motives & .78 & .59 & .21 & \\
Ready/comfortable & .12 & .05 & .01 & $.89-.95(.93)$ \\
Social standing & .01 & .07 & .03 & $.78-.90(.84)$ \\
Personality & .11 & .12 & .06 & $.87-.95(.91)$ \\
Relationship Status & .27 & .10 & .07 & $.87-.91(.89)$ \\
Romantic Relationship Characteristics & .39 & .14 & .07 & $.90-.93(.92)$ \\
Emotional Investment & .56 & .38 & .14 & $.91-.93(.92)$ \\
\hline
\end{tabular}




\section{Table 2}

Proportions of Sample Attributing Male- and Female-Stereotyped Sexual Motives for Self, Female Peers, and Male Peers, by Gender

\begin{tabular}{lccc}
\hline & Self & Female peer & Male peer \\
\hline Male-stereotyped motives & & & \\
Men & .23 & .32 & .53 \\
Women & .02 & .26 & .48 \\
Female-stereotyped motives & & & \\
Men & .68 & .56 & .17 \\
$\quad$ Women & .88 & .62 & .25 \\
\hline
\end{tabular}

